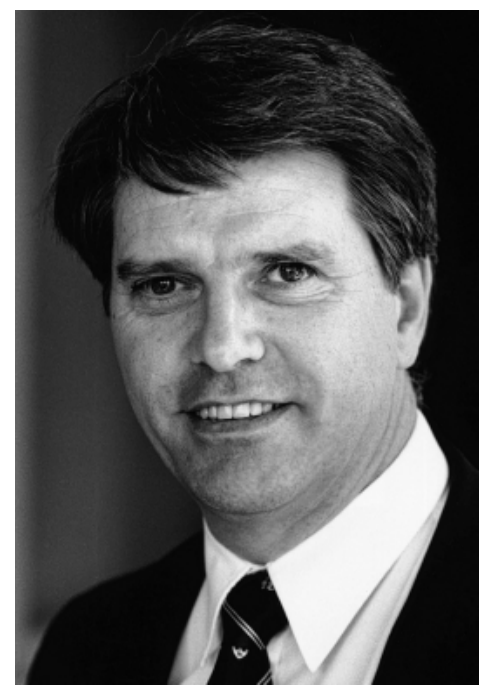

\title{
Ja zu den bilateralen Verträgen!
}

\author{
Y. Guisan \\ Korrespondenz: \\ Dr. med. Yves Guisan \\ Vizepräsident der FMH \\ Nationalrat FDP \\ Hôpital du Pays d'Enhaut \\ CH-1837 Château-d'Oex
}

Ein Teil der unter das Referendum gesetzten Unterschriften gegen die bilateralen Verträge stammt aus dem medizinischen Umfeld. Gewisse Ärztinnen und Ärzte scheinen allen ernstes zu fürchten, dass ihre europäischen Kolleginnen und Kollegen sich in rauhen Mengen in unserem Land als Konkurrenz niederlassen werden. Diese Ängste sind unbegründet, wie die fast zehnjährige Erfahrung innerhalb der EU bestätigt. Ausserdem haben die eidgenössischen Räte vorsichtshalber restriktive Massnahmen beschlossen.

Der Bundesrat wird die Anzahl der Ärztinnen und Ärzte, die auf Rechnung der obligatorischen Sozialversicherung praktizieren, dann einschränken können, wenn bewiesen ist, dass eine Ausdehnung der Gesundheitskosten zur Hauptsache auf der ärztlichen Plethora beruht. Diese Massnahme betrifft, weil jede diskriminierende Bestimmung gegen die europäischen Prinzipien verstossen würde, die Schweizer wie auch ihre Kollegen aus der EU. Aber sie wird auf einen Zeitraum von drei Jahren beschränkt. Auch wenn die Berufsausübungsbewilligung, sobald die nötigen Voraussetzungen (Anerkennung der Diplome) erfüllt sind, im Prinzip nicht mehr verweigert werden darf, hindert nichts den Bundesrat oder die Kantone daran, bezüglich Zulassung zur sozialen Krankenversicherung restriktive Massnahmen zu erlassen. Dies ist übrigens in der ganzen EU üblich. Es ist möglich, sich überall niederzulassen, aber die Zulassung zur Sozialversicherung stösst auf diverse administrative Hindernisse. Haupthindernis ist die Zahl der verfügbaren Stellen, für die es angesichts der grassierenden Arbeitslosigkeit immer Dutzende von Kandidaten hat.

\section{Schwierigkeiten bei den Bankkrediten}

Unabhängig von den rechtlichen Bestimmungen und der Freizügigkeit der Personen gibt es zahlreiche Hindernisse für eine Praxiseröffnung - auch für die jun- gen Ärztinnen und Ärzte, die sich momentan in der Weiterbildung an unseren Spitälern befinden. Zurzeit gibt es mehr als 2000 von ihnen in unserem Land. Ausser sie hätten einen gewissen Bekanntheitsgrad aufgrund einer mehrjährigen, verantwortungsvollen Tätigkeit im Spital, werden sie auf die selben finanziellen Schwierigkeiten stossen wie ihre Kolleginnen und Kollegen, die direkt aus dem Ausland kommen: Sie werden die Lokalitäten und die Einrichtung in Schweizer Franken bezahlen müssen, ebenso die Löhne des Personals. Ausserdem muss ein Patientenstamm gefunden werden, was angesichts nicht vorhandener Beziehungen nicht leicht sein wird. Kommt dazu, dass der Markt in den grösseren Städten und Ortschaften, aber auch in der Mehrzahl der Fachrichtungen weitgehend gesättigt ist. Unter diesen Umständen ist es nicht leicht, einen Investitionskredit der Banken sowie einen Betriebskredit für die ersten zwei, drei, vielleicht sogar mehr Jahre zu bekommen. Schon heute verlangen die Banken solide Garantien für derartige Kredite, die mit einigen Risiken behaftet sind.

\section{Die gegenseitige Anerkennung der Diplome erleichtert die Rückkehr ins eigene Land}

Man wird nicht erwarten müssen, dass sich die Mehrheit der europäischen Assistenz- und Oberärzte in der Schweiz niederlassen wird. Die bilateralen Verträge haben den Vorteil, dass die Aus- und Weiterbildung, die in der Schweiz gemacht wird, durch die EU anerkannt sein wird, was die Rückkehr ins Heimatland unter Umständen erleichtert. Heute blieb oftmals kein anderer Ausweg, als die schweizerische Staatsbürgerschaft zu erwerben oder sich durch Anerkennung der Weiterbildung eine Praxisbewilligung zu beschaffen - etwas, was in Zukunft wohlverstanden nicht mehr möglich sein wird. Die Gesamtheit aller rechtlichen und administrativen Vorschriften sollten unsere Kolleginnen und Kollegen, namentlich diejenigen in den Grenzregionen, beruhigen. 


\section{Die Plethora ist ein Problem, das mit den bilateralen Verträgen nichts zu tun hat}

Die Plethora ist ein Problem, das den Zentralvorstand stark beschäftigt. Bis heute war kein politischer Wille vorhanden, der es erlaubt hätte, dieses Problem grundsätzlich anzugehen. Die durch die bilateralen Verträge möglichen Konsequenzen haben ohne Zweifel zur Sensibilisierung beigetragen. Während der Zugang zum Medizinstudium heute nur per Test geregelt wird, wird die Weiterbildungsphase von weit grösseren Veränderungen betroffen. Die Qualitätsanforderungen und die Akkreditierungen werden zweifellos zu einer Verringerung der Stellenzahl führen. Die Spitalplanung wird darüber hinaus zusätzliche Stellen abbauen. Eine Arbeitsgruppe, in der neben der FMH H+ sowie die Sanitätsdirektorenkonferenz vertreten sind, studiert zurzeit die damit verbundenen Fragen. Die weitere Entwicklung wird sicherlich in Richtung mehr Vollzeitstellen und weniger Weiterbildungsstellen an den Spitälern gehen. Dies führt zu einer gewissen - legitimen - Beunruhigung unserer jungen Kolleginnen und Kollegen. Aber auch sie haben kein Interesse an einem grösseren Ungleichgewicht zwischen Weiterbildungsstellen und den späteren Möglichkeiten zur Praxiseröffnung.

\section{Die Beziehung zwischen Arzt und Patient hat starke kulturelle Bindungen}

In Europa zählt man ungefähr eine Million Ärztinnen und Ärzte. Die Erfahrungen mit der Personenfreizügigkeit innerhalb der EU hat gezeigt, dass trotz einigen tausend arbeitslosen Ärztinnen und Ärzten in den meisten Mitgliedsländern keine grosse Völkerwanderung auf der Suche nach besseren Bedingungen stattgefunden hat. Die Beziehung zwischen Arzt und Patient hat eben eine starke kulturelle Bindung. Einzig etwa 60000 Mediziner haben ihr Heimatland verlassen - in der Regel, um sich anlässlich ihrer Pensionierung an den Gestaden des Mittelmeers anzusiedeln und ohne sich auf Kosten der Sozialversicherung zu betätigen. In den meisten Fällen beschränken sie sich auf eine Privatpraxis, in der sie sich um Landsleute am Ort kümmern.

\section{Die Attraktivität der Schweiz - bitte keine Nabel- schau!}

Die Mehrheit der Unterschriften aus ärztlichen Kreisen stammt aus Regionen, in denen bereits ein starker Euroskeptizismus festzustellen ist. Es ist dabei durchaus möglich, dass sich die Ärzteschaft in erster Linie solidarisch mit den Befürchtungen ihrer $\mathrm{Pa}-$ tientinnen und Patienten erklärt und ebenfalls der Überzeugung anhängt, die Schweiz sei per definitionem attraktiv. Dies ist, wie man sich vorstellen kann, nicht mehr der Fall. Die steuerlichen Vorteile sind mehr und mehr relativ. Die bürokratischen Hindernisse beim Bau und bei der industriellen und kommerziellen Entwicklung sind gewichtig. Die Aufrechterhaltung der Prosperität beruht mehrheitlich auf dem Bewahren des Erreichten als auf der Schaffung neuer Unternehmen.

Die Einführung des Euro zum Beispiel hat nicht dazu geführt, dass vermehrt Fluchtgeld in die Schweiz gekommen ist, wie dies viele prophezeit hatten. Die Zeiten haben sich in der Tat geändert. Die Nabelschau war niemals Quelle wirtschaftlicher Entwicklung. Wir gewinnen nur einen beschränkten Vorteil aus der gegenwärtigen Wiederankurbelung der Konjunktur, weil uns der Geist der Offenheit fehlt, zu dem unabdingbar die bilateralen Verträge gehören.

\section{Die schweizerische Ärzteschaft hat auch Interesse an den bilateralen Verträgen}

Von der Ausweitung der Kontakte profitieren alle. $\mathrm{Zu}$ allen Zeiten haben die Studenten das Bedürfnis verspürt, aus unseren Mauern auszubrechen und neue Horizonte zu entdecken - aber nur schwer war dies für eine Zeit möglich, die einen eng begrenzten Stage überschritt. Selbst Weiterbildungstitel, die an renommierten ausländischen Universitäten erworben wurden, sind nicht in jedem Fall anerkannt worden. Unsere künftigen jungen Kolleginnen und Kollegen werden sich an den Universitäten der EU ausbilden, ohne dass sie auf Schwierigkeiten mit der Anerkennung ihrer Diplome stossen werden, wie dies heute der Fall ist. Die Personenfreizügigkeit öffnet den Schweizerinnen und Schweizern auch die Möglichkeit, eine Vielzahl von vor allem wissenschaftlichen und akademischen Karrieren in den EU-Ländern zu ergreifen - etwas, was bis heute nur den Cracks einer international bekannten Spezialität möglich war. Ausserdem werden sowohl die Ärzteschaft als auch die Pflegeberufe vom allgemeinen Wohlstand profitieren. Sie haben, wie der Rest der Bevölkerung auch, ein Interesse an der Normalisierung unserer wirtschaftlichen Beziehungen mit der EU und an der Eröffnung neuer Perspektiven. 